\title{
Considerações Sobre o Plano de Combate à Fome e à Miséria
}

\section{On The Plan to Combat Hunger and Misery}

\author{
Gilza S. Pereira ${ }^{1}$ \& Inês R. R. de Castro²
}

PEREIRA, G. S. \& CASTRO, I. R. R. On The Plan to Combat Hunger and Misery. Cad. Saúde Públ., Rio de Janeiro, 9 (supplement 1): 106-113, 1993.

This paper discusses the "Plan Against Hunger and Misery" as it relates to the Ministry of Health. The Plan centers around the program "Milk is Health", subsidized by the Brazilian Nutritional Surveillance System and aimed at undernourished children, his/her close relatives and pregnant women under nutritional risks identified in the public health sector. The authors raise questions regarding the role(s) to be played by the health system and by the Nutritional Surveillance System in the implementation of the Plan, the guidelines for food distribution, the indicators for selecting target groups and the parameters for evaluating the program "Milk is Health" itself. It is also argued that the Plan should value structural modifications, without which it will not be possible to ameliorate misery and hunger in Brazil.

Key words: Food Policies; Nutritional Supplementation; Nutritional Surveillance; Health System; Maternal-Infant Health

\section{INTRODUÇÃO}

A fome e a desnutrição não são companheiras recentes do povo brasileiro. Em 1946, a fome endêmica foi mapeada por Josué de Castro, dando origem ao clássico "Geografia da Fome" (Castro, 1948). Nesta mesma linha, hoje nos deparamos com o número inadmissível de 32 milhões de indigentes, evidenciado no Mapa da Fome, elaborado pelo Instituto de Pesquisa Econômica Aplicada - IPEA (Peliano, 1993). Tomando este Mapa como subsídio, o governo federal elaborou o Plano de Combate à Fome e à Miséria - Princípios, Prioridades e Mapa das Ações do Governo (Comissão Especial, 1993), (Plano) com o intuito de sistematizar, para os diversos ministérios, propostas de intervenção com vistas à erradicação da fome e da miséria.

Este ensaio pretende contribuir para o debate

\footnotetext{
${ }^{1}$ Centro de Ciências Biológicas e da Saúde da Universidade Gama Filho. Rua Manoel Vitorino, 625 Prédio AG, $4^{\circ}$ andar. Rio de Janeiro, RJ, 20748-900, Brasil.

${ }^{2}$ Secretaria Municipal de Saúde do Rio de Janeiro. Rua Afonso Cavalcanti, 455/823. Rio de Janeiro, RJ, 20211-110, Brasil.
}

sobre a estruturação das medidas propostas pelo governo federal, particularmente em relação ao Ministério da Saúde (MS). O Plano é inicialmente apresentado e seus pontos são discutidos detalhadamente, à luz da literatura científica e da experiência histórica da distribuição de alimentos pelo setor saúde.

Espera-se que as reflexões aqui apresentadas possam contribuir para que o Plano seja executado de forma a garantir, de fato, condições dignas de vida para o povo brasileiro.

\section{AS AÇÕES PREVISTAS PELO PLANO PARA O MINISTÉRIO DA SAÚDE}

O Plano apresenta como objetivos do MS: (a) "recuperar crianças desnutridas e reduzir a prevalência das diversas formas de desnutrição" e (b) "consolidar e assegurar a prestação de ações básicas de saúde, em observância aos princípios do Sistema Único de Saúde” (p. 17).

Nos mecanismos operacionais para a obtenção destes objetivos está prevista uma única intervenção: o Programa "Leite é Saúde", a ser desenvolvido através do Sistema Único de Saúde, atendendo crianças menores de 2 anos (e 
seus contatos familiares) e gestantes de risco nutricional. O Programa apresenta as seguintes características: (a) "distribuição de alimentos (leite e óleo de soja), associada à prestação das ações básicas de saúde, entre as quais se destacam: acompanhamento do crescimento $e$ desenvolvimento da criança, consulta clínica individual e imunização; atenção a gestantes, especialmente no que se refere à avaliação do crescimento intra-uterino, consulta clínica individual, identificação e tratamento das patologias intercorrentes, orientação e educação em saúde"; (b) "execução descentralizada, incluindo a aquisição dos alimentos, que será realizada pelos próprios municípios que aderirem ao Programa”; (c) "seleção dos municípios que optarem pelo atendimento integrado ao desnutrido entre os que já tenham implantado um Sistema de Vigilância Alimentar e Nutricional - Sisvan"; e (d) "implantação do Programa de forma gradual, iniciando-se no segundo semestre de 1993" (p. 17).

Propõe-se o início da implantação do Programa para o segundo semestre de 1993, quando serão atendidos $20 \%$ da clientela-alvo, estando a universalização do atendimento prevista para o primeiro semestre de 1995.

\section{Sobre as Ações Previstas para o Ministério da Saúde}

\section{Sobre o Papel do Setor Saúde}

Embora os objetivos propostos para o MS no Plano contemplem, a nosso ver, a vocação da Saúde - assistir à população, promovendo saúde, prevenindo e curando agravos —, não vislumbramos mecanismos operacionais que dêem conta da mesma. Pelo contrário, a intervenção valorizada foi a de distribuição de alimentos, que, como discutiremos adiante, não nos parece reforçar esta vocação.

Analisando os determinantes imediatos, mediatos e estruturais do perfil nutricional da nossa população, podem ser observados dois eixos claros. O primeiro diz respeito ao consumo de alimentos, que envolve, entre outros fatores, a produção, comercialização, poder de compra e distribuição intrafamiliar dos alimentos. O segundo refere-se à utilização biológica dos alimentos, que envolve, entre outros fato- res, as condições de moradia, a ocorrência de agravos infecciosos ou não e o acesso aos serviços de saúde (Beghin, 1988). A nosso ver, o papel do setor saúde é concentrar esforços neste último eixo, investindo em atividades educativas e contribuindo para que, através de um atendimento diferenciado para os grupos sob risco nutricional, o ciclo vicioso "infecções-desnutrição" não se instale ou, pelo menos, não se agrave.

Embora saibamos que estas medidas são limitadas em termos de "resolução" da questão alimentar e nutricional, entendemos ser esta a contribuição da Saúde no enfrentamento e superação desta complexa questão, que só pode ser encarada numa perspectiva intersetorial.

No entanto, não é inédita a proposta de distribuição de alimentos via MS, distorcendo seu papel. Outra distorção - também histórica - prevista no Plano é o papel conferido ao Sisvan e sua relação com o MS. Ambas serão discutidas a seguir.

\section{Sobre o Papel Conferido ao Sisvan}

Data de 1974 o primeiro registro oficial do conceito de Vigilância Nutricional (VN), formulado na Conferência Mundial de Alimentação, fazendo-se uma transposição do conceito de vigilância das enfermidades, amplamente aceito à época. Vinculada à criação de um programa global de Segurança Alimentar (SA), a VN operacionalizou-se no Sisvan (Macias et al., 1980; Mason et al., 1984).

A SA sistematiza o que é desejável de ser alcançado pelos países em geral em termos de alimentação e tem como objetivo final assegurar, a todo o momento e a toda a população, a possibilidade material e econômica de obter os alimentos básicos de que necessita. Seus objetivos específicos são assegurar a produção de alimentos em quantidades suficiente, conseguir a máxima estabilidade dos subsídios de tais alimentos e garantir, àqueles que necessitam, a possibilidade de obtenção dos alimentos (Opas, 1989).

O Sisvan foi proposto como um instrumento fundamental para o conhecimento da situação alimentar e nutricional de uma população, contribuindo para a construção da SA. Ele consiste num sistema de coleta, processamento 
e análise contínuas dos dados de uma população, possibilitando um diagnóstico atualizado da situação nutricional e de suas tendências temporais. Contribui para que se conheçam a natureza e magnitude dos problemas de nutrição, caracterizando grupos sociais de risco e dando subsídios para a formulação de políticas, estabelecimento de programas e intervenções (Mason et al., 1984; Batista Filho, 1985; Opas, 1989). Para que o Sisvan viabilize-se plenamente, vários setores devem estar envolvidos. Dentre estes, os mais importantes são: Agricultura, Economia, Planejamento, Educação e Saúde, cabendo a este último a produção de dados sobre o perfil nutricional da população.

A nosso ver, esta dimensão ampla do Sisvan não está presente no Plano. Ao contrário, este sistema só está previsto nas propostas para o MS, servindo como instrumento de uma única medida de construção da SA, justamente uma das mais polêmicas: a suplementação alimentar.

Vincular o Sisvan, na rede de saúde, à suplementação alimentar, não valorizando seu papel como um sistema que subsidia o planejamento e a avaliação de políticas e intervenções que sejam da vocação do próprio Sistema de Saúde - fundamentalmente, a de melhoria da assistência por ele prestada - é não valorizar o segundo objetivo previsto pelo Plano para o MS.

Além disso, não resgatar, nesta conjuntura política, o papel de articulação intersetorial do Sisvan, subsidiando e avaliando políticas mais estratégicas que a suplementação alimentar, é deixar de investir em mais uma frente de resgate do compromisso dos organismos governamentais com a SA e, portanto, com a construção da cidadania.

\section{Sobre a Ação Específica de Distribuição de Alimentos}

Inicialmente denominado Programa de Nutrição em Saúde (PNS), o II Programa Nacional de Alimentação e Nutrição (Pronan), instituído pelo Instituto Nacional de Alimentação e Nutrição (Inan) em 1976 (Inan, 1976), propunha como principal linha de atuação a suplementação alimentar. Portanto, a proposta de se promover uma suplementação alimentar para grupos vulneráveis já foi vivenciada no setor saúde. Segundo avaliação feita através da Pesquisa Nacional Sobre Saúde e Nutrição (PNSN) em 1989, os programas federais de suplementação alimentar apresentam baixa cobertura e não atingem os mais necessitados (Inan/Fibge/IPEA, 1990). Ou seja, não é possível afirmar que a suplementação alimentar tenha tido real impacto no perfil nutricional brasileiro. Em contrapartida, os problemas vinculados a este Programa foram inegavelmente importantes.

A vinculação das ações básicas de saúde à distribuição de alimentos, se, por um lado, pode aumentar a freqüência da população à rede básica de assistência à saúde, por outro pode servir para distorcer e descaracterizar a importância das ações básicas enquanto prevenção de doenças e promoção da saúde, deslocando a compreensão da população para as ações como mediadoras da oferta de uma sacola de alimentos. Desta forma, não havendo mais o alimento, as ações básicas "perdem seu valor" para a população.

Além disso, a operacionalização de programas de suplementação dentro dos serviços de saúde representa, para estes, um fator complicador. Os postos e centros de saúde não possuem infra-estrutura que permita a estocagem de alimentos para posterior distribuição. Faltam espaço adequado e recursos humanos para lidar com a guarda e distribuição de gêneros alimentícios. Outro aspecto relevante é o risco concreto de desvio dos mesmos, pois a população entende que os produtos destinam-se aos "mais pobres", por mais que se afinem os critérios de seleção dos beneficiários, através da inclusão de indicadores biológicos. Como disse L'Abbate (1989), a vulnerabilidade biológica, na verdade, esconde a vulnerabilidade social, e é esta, sim, que precisa ser atacada e revertida.

Fora estas questões de infra-estrutura local da rede básica de saúde, foi evidente a utilização política das medidas de suplementação alimentar. Peliano (1988) afirma que o PNS foi "mиitas vezes utilizado como um instrumento de manipulação política". Entendemos o uso político não apenas no seu sentido macro, ao reforçar a dependência do clientelismo estatal, mas também no sentido das micro-agências de 
poder locais, representadas, em especial, pelas associações de moradores e pelas próprias unidades de saúde.

Essa problemática foi vivida durante quase uma década pelos profissionais da rede de saúde e, independentemente de uma avaliação a nível nacional, há a avaliação pessoal de cada ator social. E aqui não nos referimos apenas aos profissionais de saúde, mas à própria população "beneficiada". Por exemplo, entre as causas do abandono do PNS em Osasco, São Paulo, $19,8 \%$ dos motivos citados pelos beneficiários era a má qualidade dos alimentos (Kalil, 1984).

Diante de tais questões, é evidente que a perspectiva de retomar um programa de suplementação alimentar via unidades da rede básica de saúde nos assusta. No nosso entender, os problemas vivenciados na execução de programas de suplementação alimentar fatalmente se repetirão em qualquer iniciativa que utilize a distribuição de alimentos como resposta governamental à questão alimentar e nutricional. Além disso, é fundamental levar em conta que boa parcela dos indigentes sequer chega aos serviços de saúde (Peliano, 1988).

É claro que, neste momento em que a fome aflige de forma avassaladora um enorme contingente de nossa população, de maneira alguma somos contra a utilização de medidas emergenciais que venham atender de imediato às necessidades nutricionais dos grupos de maior risco. Acreditamos, porém, que sejam possíveis medidas emergenciais, que não a suplementação alimentar, que garantam maior acesso aos alimentos por parte da população menos favorecida, através, por exemplo, de sacolões itinerantes que comercializem produtos subsidiados dentro dos bolsões de pobreza, e não só na rede formal de abastecimento.

\section{Sobre o Programa "Leite é Saúde"}

Serão levantados, a seguir, alguns aspectos que dizem respeito a pontos específicos do Programa "Leite é Saúde" que não estão claros, que não foram contemplados no Plano ou que, a nosso ver, merecem maior reflexão.

\section{Indicadores e Pontos de Corte}

Nos mecanismos operacionais fala-se em atendimento integral ao desnutrido, vinculado ao Sisvan, mas não são explicitados os parâmetros a serem utilizados.

$\mathrm{O}$ instrumento de monitoramento do crescimento de crianças menores de 5 anos preconizado pelo MS - o Cartão da Criança — utiliza o indicador peso/idade e, como ponto crítico, o percentil 10 da população de referência do NCHS (MS/Inan/Inamps, 1984; NCHS, 1977). Além deste percentil, o Inan sugere, para fins de VN, a utilização do percentil 3 (MS/Inan, 1991b), que indicaria as crianças sob maior risco nutricional. A nosso ver, a inclusão da curva referente a este percentil no gráfico do Cartão da Criança é fundamental, pois, sem criar qualquer instrumento paralelo ao Cartão (pelo contrário, valorizando-o), permite selecionar crianças sob maior risco e que, portanto, devem ter prioridade no atendimento (Castro \& Anjos, 1993).

No que tange às gestantes, imaginamos que o indicador a ser utilizado seja peso/estatura segundo semana gestacional, tomando como ponto de corte a linha inferior do gráfico de Rosso (Rosso, 1985), abaixo da qual localizam-se as gestantes com risco de darem à luz filhos com baixo peso ao nascer. Sabemos que é polêmica a utilização deste gráfico, uma vez que parece haver superestimação na predição de risco de baixo peso, particularmente em relação às gestantes adolescentes, grupo em que o instrumento vem também sendo utilizado, embora não tenha sido criado para tal. Alguns estudos vêm sendo realizados com o intuito de validar este instrumento para a população brasileira, e, ainda que não estejam disponíveis resultados conclusivos, é este o instrumento preconizado pelo MS para o monitoramento do ganho de peso no pré-natal e pelo Inan para fins de VN da gestante (MS/Inan, 1991b).

\section{Sisvan Implantado como Pré-requisito para o Programa}

Ainda nos mecanismos operacionais, é dito que os municípios serão selecionados "entre os 
que já tenham implantado o Sisvan". Este requisito merece uma reavaliação em relação a dois aspectos. $\mathrm{O}$ primeiro diz respeito ao fato de as maiores prevalências de desnutrição ocorrerem nas regiões Nordeste e Norte, particularmente no meio rural (Inan/Fibge/IPEA, 1989). Neste sentido, seria interessante planejar estratégias que abranjam também os municípios que não possuam sistema de saúde ou Sisvan organizados e que necessitem de uma atenção mais efetiva. $\mathrm{O}$ segundo aspecto diz respeito à implantação propriamente dita do Sisvan na rede de saúde. Sabendo-se que este processo é gradual e complexo, cabe a pergunta: o que será considerado como "Sisvan implantado"? Isto é, quais os mínimos de cobertura (percentual de unidades com o sistema implantado) e de estruturação do fluxo de informações que serão aceitáveis?

\section{Tipo de Alimento Distribuído}

A decisão sobre qual(is) alimento(s) a ser(em) distribuído(s) em qualquer programa de suplementação alimentar é sempre um ponto extremamente polêmico que deve ser muito bem fundamentado. A escolha do leite a princípio pode parecer interessante, pois, além de ser menos vulnerável à distribuição intrafamiliar, por ser visto como um alimento infantil, o leite é de difícil acesso, por ser caro. Outro aspecto é que, sendo fornecido em pó, o leite não depende de refrigerador para ser conservado, sendo de fácil armazenamento.

No entanto, sabemos que há vários aspectos que depõem contra a utilização deste alimento. Por exemplo, o fornecimento do leite em pó implica a adição de água, que muitas vezes não está disponível ou com níveis de qualidade aceitáveis para grande parte da população-alvo do programa. Portanto, a água necessária para a ingestão do alimento fornecido pode veicular uma infinidade de microorganismos causadores de infecções extremamente espoliadoras. Além disso, embora esteja prevista a descentralização na compra do alimento, a escolha de leite em pó exclui inúmeras empresas de pequeno e médio portes que fabricam leite, mas só leite fluido, as quais poderiam ser beneficiadas indiretamente pelo programa, através da ampliação da oferta de trabalho.
Neste sentido, é fundamental que, na formulação do Plano, dentro dos princípios de solidariedade e parceria, seja levada em conta a contribuição de entidades da sociedade civil que militam nas áreas de nutrição e saúde e que vêm realizando discussões consistentes sobre este assunto.

Não ficou claro, também, se o leite a ser fornecido será integral, semidesnatado ou desnatado, nem se a adição do óleo de soja será feita no sentido de "complementar" o aporte energético do leite (se este for integral) ou de "completá-lo" (se for semi ou completamente desnatado). De qualquer forma, parece-nos bastante questionável a viabilidade do uso do óleo de soja, uma vez que este alimento está sujeito à distribuição intrafamiliar, sendo difícil garantir que ele será de fato consumido pelos mesmos indivíduos que fizerem uso do leite. Logo, seja qual for o objetivo da introdução do óleo, há grandes chances de este não ser alcançado, o que é particularmente indesejável se o leite for desnatado.

\section{Suplementação x Amamentação}

Outra questão que nos preocupa enormemente diz respeito à faixa etária das crianças a serem beneficiadas. Na faixa proposta - menores de 2 anos - estão incluídas crianças menores de 6 meses, para as quais há indicação de aleitamento exclusivo, sendo o incentivo a esta prática uma ação básica do Programa de Atenção Integral à Saúde da Criança (PAISC), normatizado pelo MS (MS/Inamps, 1986). Distribuir leite para esta faixa etária representa um recuo nas conquistas históricas de organismos governamentais e entidades da sociedade civil no sentido de promover e garantir o aleitamento materno exclusivo até o $6^{\circ}$ mês de vida (OMS/Unicef, 1989; MS/Inan, 1991a).

\section{Definição de Contatos Familiares}

Outro ponto que merece maior esclarecimento é a definição de "contatos familiares" das crianças menores de 2 anos que também seriam beneficiados pelo Programa. O que está sendo entendido como contato? Irmãos na mesma faixa etária, irmãos em qualquer idade e/ou gestantes ou crianças em qualquer idade que 
morem na mesma casa? Uma definição mais clara deste conceito trará desdobramentos na operacionalização do programa, tanto em relação à alocação de recursos quanto na organização dos serviços de saúde para o monitoramento do estado nutricional dos beneficiários.

\section{SOBRE A AVALIAÇÃO DAS MEDIDAS PROPOSTAS PARA O MINISTÉRIO DA SAÚDE}

A avaliação, em qualquer projeto ou programa, é fundamental, pois permite saber se e como os objetivos e metas foram de fato alcançados. No entanto, se analisarmos diversas propostas, projetos, programas e medidas governamentais já lançados, executados e retirados de cena nos últimos anos, percebemos ser muito recorrente a ausência de avaliação dos mesmos. Isto deixa margem para que os erros sejam repetidos e para que os acertos não sejam revivenciados.

Sentimos falta de uma preocupação com a avaliação das medidas propostas para o MS, que explicitar-se-ia na indicação de critérios claros para tal. Avaliar exclusivamente se as metas de cobertura foram alcançadas (supondo, já que isto não está enunciado no documento, que seja este o parâmetro a ser utilizado) não nos parece suficiente. Acreditamos que a elaboração e a inclusão de outros critérios (por exemplo, indicadores de operacionalização e impacto do programa) permitirão não só o amadurecimento do conteúdo das medidas em si, mas também como a ampliação da credibilidade do Plano junto à sociedade civil.

\section{SUGESTÕES}

Diante da implantação, já prevista, do Programa "Leite é Saúde", sistematizaremos, a seguir, as discussões apresentadas, entendendo que medidas emergenciais são necessárias e devem ser viabilizadas, mas com critério e consciência de sua limitação e caráter paliativo.

Em relação aos indicadores e pontos de corte, sugerimos que sejam utilizados os já preconizados pelo Inan para fins de VN (MS/Inan, 1991b): (a) o indicador peso/idade para a população infantil, sendo assumidos como pontos de corte os percentis 10 e 3, com atenção prioritária para aquelas localizadas abaixo do percentil 3, e (b) o indicador peso/estatura segundo semana gestacional, tomando como ponto de corte a linha inferior do gráfico de Rosso (Rosso, 1985).

Supondo-se que seja mantido o leite como produto a ser distribuído e conhecendo-se a grave problemática que representa a utilização de leite desnatado em um programa desta natureza, consideramos importante que: (a) a faixa etária infantil beneficiária do Programa exclua as crianças menores de 6 meses, evitando o choque com a preconização (pelo próprio MS) de promoção do aleitamento materno exclusivo até o $6^{\circ}$ mês de vida (MS/Inamps, 1986); (b) seja contemplada, no Programa, a distribuição de leite fluido nos municípios ou áreas onde isto for possível, respeitando-se o princípio da descentralização; e (c) o leite fornecido seja integral, pois, caso mantenha-se a adição de óleo, esta terá um papel complementar e, principalmente, para que seja respeitada a perspectiva de resgate da cidadania, fornecendo um alimento mais completo àqueles sob maior risco nutricional.

Quanto à distribuição dos alimentos, sugerimos que esta seja de responsabilidade de outro ministério que não o da Saúde, visto que este, como já argumentamos, não deve ter sua vocação desvirtuada por um vínculo inevitável da distribuição de alimentos com as ações básicas de saúde e o Sisvan. Esta distribuição poderia ser feita através de unidades do Ministério do Bem-Estar Social e/ou através das Forças Armadas, como previsto no Plano (p. 32). Estas últimas dispõem de recursos humanos e físicos que permitiriam melhor estocagem, transporte e distribuição dos alimentos. Além disto, sua respeitabilidade possibilitaria uma execução mais satisfatória do processo de aquisição, estocagem e distribuição dos mesmos à população beneficiária. Por fim, vincular sua presença à proposta de suplementação alimentar garantiria o caráter emergencial da medida.

Neste contexto, a rede de saúde responsabilizar-se-ia por (a) diagnosticar os grupos de risco a serem contemplados pelo Programa, referenciando-os a uma unidade de distribuição determinada; (b) acompanhar diferenciadamente os 
grupos diagnosticados; e (c) contribuir na avaliação do impacto do Programa na saúde da população beneficiada.

\section{CONSIDERAÇÕES FINAIS}

Não há dúvidas de que o enfrentamento e a superação deste quadro de fome e miséria só serão possíveis se forem assumidos os princípios de solidariedade, parceria e descentralização, previstos no Plano, e se forem também articuladas entre governo e sociedade civil medidas emergenciais, dada a magnitude do problema, mas, fundamentalmente, medidas de caráter estrutural, com impacto a médio e longo prazos.

Neste sentido, é imprescindível um investimento concreto na continuação e ampliação das ações básicas já implantadas na rede de saúde, bem como na universalização de seu acesso, elemento fundamental para a viabilização dos objetivos gerais apresentados no Plano para o MS.

Acreditamos, também, que deva ser valorizada a importância da questão alimentar e nutricional na determinação do processo saúde-doença, tanto na formação de recursos humanos na área de saúde e educação quanto nos currículos da escola básica, compreendendo $1^{\circ}$ e $2^{\circ}$ graus.

Por fim, consideramos fundamental a articulação dos Ministérios da Economia, Planejamento, Agricultura, Saúde e Educação para a estruturação do Sisvan numa perspectiva ampla, intersetorial, como preconiza a OMS, servindo como um sistema de informações que subsidie e avalie políticas e medidas (emergenciais e estruturais) voltadas para o alcance da SA. E, tendo como ponto de partida esta perspectiva, cabe ao MS e a toda a rede pública de assistência a implantação do Sisvan, entendendo que este (a) contribui para a reorganização dos serviços (através do mapeamento de grupos de risco que merecem atenção diferenciada); (b) potencializa a prática dos profissionais de saúde, uma vez que confere a dimensão coletiva (de perfil nutricional da clientela) a uma prática de acompanhamento individual; e (c) além de mapear grupos sob risco nutricional, permite o monitoramento do estado nutricional, indicador síntese da qualidade de vida, conferindo um outro enfoque à produção de estatísticas sobre indicadores de saúde, que tradicionalmente trabalham dados de morbi-mortalidade.

\section{AGRADECIMENTOS}

Registramos a participação de Elda L. Tavares (SMS/RJ), Marília B. Castello (SMS/RJ; UGF), Simone A. Serrão (UGF), Suzete M. F. Santos (SMS/RJ)), Cláudia Valéria C. da Silva (UGF; Ensp/Fiocruz) e Érica Ell (Ensp/Fiocruz) no amadurecimento deste tema e agradecemos sua colaboração na revisão do documento que deu origem a este artigo.

\section{RESUMO}

PEREIRA, G. S. \& CASTRO, I. R. R.

Considerações Sobre o Plano de Combate à Fome e à Miséria. Cad. Saúde Públ., Rio de Janeiro, 9 (suplemento 1): 106-113, 1993.

Neste trabalho discute-se a proposta do Plano de Combate à Fome e à Miséria para o Ministério da Saúde. O programa "Leite é Saúde" constitui a linha central de atuação do Plano, sendo subsidiado pelo Sistema de Vigilância Nutricional (Sisvan) e dirigido a crianças desnutridas, seus contatos familiares e gestantes sob risco nutricional atendidos na rede de saúde. São levantados questionamentos relativos aos papéis previstos para o setor saúde e para o Sisvan, à ação específica de distribuição de alimentos, aos indicadores empregados e população-alvo e aos critérios de avaliação do programa "Leite é Saúde". Os autores argumentam que o Plano deve valorizar a execução de medidas estruturais, sem as quais não será possível superar o quadro de miséria e fome que acomete segmentos da população brasileira.

Palavras-Chave: Políticas de Alimentação; Suplementação Alimentar; Vigilância Nutricional; Sistema de Saúde; Saúde Materno-Infantil 


\section{REFERÊNCIAS BIBLIOGRÁFICAS}

BATISTA-FILHO, M., 1985. Sistema de Vigilância Nutricional. Trabalho apresentado no Encontro sobre Sistema Alimentar e Nutricional na América Latina e Caribe. Santiago, Chile. (Mimeo.)

BEGHIN, I.; CAP, M. \& DUJARDIN, B., 1988. A Guide to Nutritional Assessment. Geneve: World Health Organization.

CASTRO, J., 1948. Geografia da Fome - a Fome no B rasil. Rio de Janeiro, O Cruzeiro.

CASTRO, I. R. R. \& ANJOS, L. A., 1993. Vigilância nutricional e morbidade de crianças menores de 5 anos numa unidade básica de saúde: análise da série histórica 1987-91. Cadernos de Saúde Pública, 9 (supl.1): 36-45.

COMISSÃO ESPECIAL (Comissão Especial para Propor Medidas de Combate a Fome, ao Desemprego e a Recessão), 1993. Plano de Combate à Fome e à Miséria - Princípios, Prioridades e M apa das A ções de Governo. Brasília. (Mimeo.)

INAN (Instituto Nacional de Alimentação e Nutrição), 1976. Programa Nacional de Alimentação e Nutrição - PRONAN - 1976-1979. Brasília: MS. (Documento Técnico, 6/76)

INAN (Instituto Nacional de Alimentação e Nutrição); FIBGE (Fundação Instituto Brasileiro de Geografia e Estatística) \& IPEA (Instituto de Pesquisa Econômica Aplicada), 1990. Resultados Preliminares da Pesquisa Nacional sobre Saúde e Nutrição. Brasília: MS.

KALIL, A. C., 1984. Causas do abandono do Programa de Nutrição em Saúde. A limentação e Nutrição, 5: 46-52.

L'ABBATE, S., 1989. As políticas de alimentação e nutrição no Brasil II. A partir dos anos setentas. Revista de Nutrição, 2: 7-54.

MACIAS, J. A.; DAZA, C. H. \& PRADILLA, A., 1980. Fundamentos de un sistema de vigilancia alimentaria e nutricional. Boletin de la Oficina Sanitaria Panamericana, 89: 538-545.

MASON, J. B.; HABICHT, J.; TABATABAI, H. \& VALVERDE, V., 1984. Vigilancia Nutricional. Genebra: OMS.
MS (Ministério da Saúde) \& INAMPS (Instituto Nacional de Assistência Médica da Previdência Social), 1986. A leitamento $M$ aterno e Orientação A limentar para o D esmame. Brasília: MS.

MS (Ministério da Saúde) \& INAN (Instituto Nacional de Alimentação e Nutrição), 1991a. Programa Nacional de Incentivo ao A leitamento $M$ aterno. Brasília: MS. , 1991b. Sistema de Vigilância A limentar e Nutricional - Proposta para A tuação a Nível Nacional. Brasília: MS.

MS (Ministério da Saúde); INAN (Instituto Nacional de Alimentação e Nutrição) \& INAMPS (Instituto Nacional de Assistência Médica da Previdência Social), 1984. A companhamento do Crescimento e Desenvolvimento. $3^{\mathrm{a}}$ ed., Brasília: MS.

NCHS (National Center for Health Statistics), 1977. NCHS Growth Curves for Children Birth-18 Years United States. Washington, D.C.: U.S. Printing Office. (Vital and Health Statistics Series 11, no. 165, DHEW Pub. no. 78-1650).

OMS (Organização Mundial de Saúde) \& UNICEF (Fundo das Nações Unidas para a Infância), 1989. Proteção, Promoção e A poio ao Aleitamento Materno: o Papel Especial dos Serviços $M$ aterno-Infantis. Genebra: OMS.

OPAS (Organização Panamericana de Saúde), 1989. Vigilancia Alimentaria y Nutricional en las A mericas. Washington, D.C.: Organización Panamericana de la Salud/Organización Mundial de la Salud.

PELIANO, A. M. T. M., 1988. Os programas alimentares e nutricionais no contexto da recessão econômica: 1980-1984. In: Crise e Infância no Brasil: o Impacto das Políticas de Ajustamento Econômico (J. P. Chadad \& R. Cervini, orgs.), pp. 185-220, São Paulo: Unicef/IPE-USP.

(Org.), 1993. 0 M apa da Fome - Subsídios à Formulação de uma Política de Segurança Alimentar. Brasília: Instituto de Pesquisa Econômica Aplicada.

ROSSO, P. A., 1985. New chart to monitor weight gain during pregnancy. A merican Journal of Clinical Nutrition, 41: 644-652. 